\title{
Speed Control and Power Factor Improvement of a Single Phase AC Motor
}

\author{
Aamir Hayat ${ }^{1}$, Muhammad Nasir Khan ${ }^{2, *}$ \\ ${ }^{1}$ Faculty of Electrical Engineering, University of Central Punjab, 1 - Khayaban-e-Jinnah Road, Johar Town, Lahore \\ ${ }^{2}$ Department of Electrical Engineering, The University of Lahore, Lahore, Pakistan \\ *Corresponding Author: khamy018@mymail.unisa.edu.au
}

Copyright (C) 2014 Horizon Research Publishing All rights reserved.

\begin{abstract}
Low power factor is usually not a big problem in residential homes. It does, however, become a problem in industries where multiple motors are operational. Therefore, there is a dire requirement to correct the power factor in industries. To rectify the issue and providing a fruitful solution is the major task of our research work. Although there are different methods of power corrections are available with certain pros and cons. Occasionally, the power factor correction capacitors are implemented to correct the problems using pacific techniques and somehow active techniques as well. Each technique has its own limitations in terms of computational complexity and implementation cost. In the proposed technique, we implement the micro-controller and programmable logic controller based system in combination with capacitor bank for the power factor correction. This way, we can efficiently control the power factor.
\end{abstract}

Keywords Programmable Logic Controller (PLC), Power Factor Correction (PFC), Induction Motor (IM), Zero Crossing Detector (ZCD)

\section{Introduction}

The use of alternating current (AC) induction motor is usually essential in industries and other residential utilities. AC induction motors consume large percentage of energy (i.e., more than 50 percent of the energy used in industry) [1]. The power factor of the induction motor is relatively poor as compared to other types of load, causing higher line currents, with additional heat in line cables and transformers. A poor power factor is mostly the results of large voltage and current phase difference. The other reasons include the high harmonic contents and distorted current waveform. Most probably the poor power factor is also the result of inductive loads, i.e., induction motor [2]. Low power factor is usually not a big problem in residential homes. It does however become a problem in industry where multiple motors are used. Therefore, power factor correction (PFC) needs to be done in industries [3]. A poor power factor due to inductive loads can be improved by adding the PFC circuit [4].

The ideal figure for load power factor is one (i.e., a purely resistive load), because it requires a very small amount of current to transmit a given real power [5]. Real loads deviate from this ideal condition. Electric motor loads are phase lagging (inductive), therefore, requiring capacitor banks to counter this lagging issue. Electricity utilities measure reactive power used by high demand customers and charge higher rates accordingly. Some consumers install power factor correction schemes in factories to cut down the higher costs [6].

From the discussion in [7-10], we came to know that the PFC is desirable because the source of electrical energy must be capable of supplying real power as well as any reactive power demanded by the load. The procedure of PFC requires larger and more expensive power plant equipment, transmission lines, and transformers and switches [5]. Resistive losses in transmission lines waste some of the generated power because the extra current is needed to supply the reactive power, which only serves to heat up power lines $[11,12]$.

The PFC is achieved by different methods including the passive, active, synchronous condenser and capacitor bank based systems [13-16]. Each method has its own limitations with possible advantages, depending on the desired application. In our proposed technique, we suggest PFC using a microcontroller, programmable logic controller (PLC) and capacitor bank based system. By using the proposed technique, we can improve the efficiency and system and electricity consumption cost as well. The proposed work focuses on the implementation of a laboratory model for PLC based PFC to improve the power factor of single-phase induction motor. During the online process a set of capacitors sized in a binary ratio was switched ON or OFF with the help of zero voltage static switches according to a control strategy to obtain a pre-specified power factor.

The proposed control strategy relies on a look-up table and an intelligent system. The look-up table is prepared according to a measured value of the phase angle between the stator phase voltage and the stator phase current. The 
design aims to monitor the phase angle continuously and in the event of phase angle deviation, a correction action is initialized to compensate the difference by continuous changing variable capacitor value via a switching process. The motor was used as a medium to show that the power factor can be controlled using the PLC. In the proposed method, the PLC senses any changes occur in the speed of motor. Then, accordingly, it turns ON or OFF capacitors in the capacitor bank (i.e., depending on the speed of motor either increasing or decreasing). In this way, we maintain the power factor value at a desired level. The power factor remains same no matter how many motors turned ON or OFF. The overall system requires only one microcontroller chip, some power electronic components, capacitor bank and a PLC unit for switching purpose.

Our motivation in this paper is to reduce the power theft and provide a reliable meter reading system. By providing the proposed AMI system, we can improve the current metering system and able to collect the reliable meter data. We can then certainly reduce the power theft, improve the billing system, manage the grid and can point-out the areas with high line losses. There are several models of AMI system implemented in various parts of the world. These models of AMI system are categorized based on the communication between the meter and the central management system. Each model has its own technical and physical limitations. The AMI system model at one place hardly implemented to any other place with same settings. We have to select the model; have to modify it so that it can meet the challenges of the current system implementation, which include the technical, legal, managerial and economical issues in Pakistan.

\section{System Modelling}

This project is composed of a motor controller and a power factor correction controller for a single-phase induction motor (IM), utilizing the programmable logic controller (PLC). During the online process a set of capacitors sized in a binary ratio will be switched $\mathrm{ON}$ or OFF with the help of zero voltage static switches according to a control strategy to obtain a pre-specified power factor. This control strategy relies on a lookup table as described in Section I. The speed of the motor is controlled by the VSD (variable speed drive). This is used to control the rotational speed of the $\mathrm{AC}$ electric motor by controlling the frequency of the electrical power supplied to the motor.

Speed of the motor changes depending on variable load. As the load varies the power factor also changes. This change in power factor is detected by PLC, which improve the value of power factor to the desired value. For a DC circuit the power is $\mathrm{P}=\mathrm{VI}$, and this relationship also holds for the instantaneous power in an AC circuit. However, the average power in an $\mathrm{AC}$ circuit expressed in terms of the rms voltage and current is [3]

$$
P_{\text {avg }}=\operatorname{VIcos}(\varphi)
$$

where $\varphi$ is the phase angle between the voltage (V) and current (I). The additional term is called the power factor. The voltage and current is given by [5],

$$
\begin{gathered}
\mathrm{P}_{\mathrm{avg}}=\operatorname{VI} \cos (\varphi) \quad V=V_{m} \sin (\omega t) \\
I=I_{m} \sin (\omega t-\varphi)
\end{gathered}
$$

Then the instantaneous power at any time is given by,

$$
P_{\text {ins }}=V_{m} I_{m} \sin (\omega t) \sin (\omega t-\varphi)
$$

Using the trigonometric identities, (4) can be further expanded to,

$P_{\text {ins }}=$

$V_{m} I_{m} \sin (2 \omega t) \cos (\varphi)-V_{m} I_{m} \sin (\omega t) \sin (\varphi) \cos (\omega t)$

Generally, four types of techniques are used for power factor correction, passive, active, synchronous and capacitive. Each method has its own pros and cons. Passive PFC is very simple but it causes harmonic, which makes this method not useful. Capacitive PFC is better for circuits that use IM as a means of reducing the inductive component of the current and thereby reduce the losses in the supply. Power factor correction is achieved by the addition of capacitors in parallel with the connected motor circuits and can be applied at the starter, or applied at the switchboard or distribution panel. The resulting capacitive current is leading current and is used to cancel the lagging inductive current flowing from the supply [6]. The main diagram of our project is shown in Fig. 1.

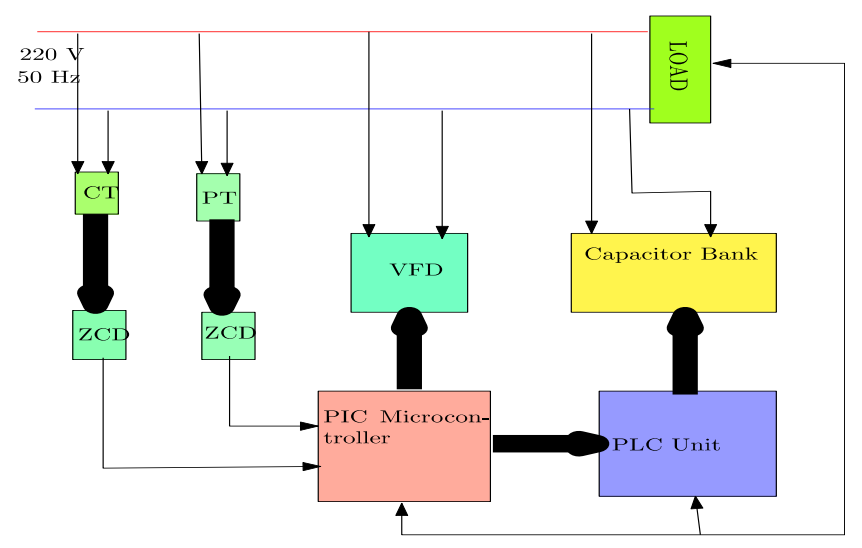

Figure 1. Proposed system block diagram.

The design aims to monitor the phase angle continuously and in the event of phase angle deviation, a correction action is initialized to compensate for this difference by continuous changing variable capacitors value via switching process. The overall system requires only one PIC chip, a few power electronic components and a bank of capacitors and PLC unit for switching the capacitor banks. PFC is a technique of counteracting the undesirable effects of electric loads that create a power factor that is less than one. PFC may be applied either by an electrical power transmission utility to improve the stability and efficiency of the transmission network or correction may be installed by individual electrical customers to reduce the costs charged to them by their electricity supplier $[3,4,13]$. This paper defines the 
programmable interface controller (PIC) and PLC based power factor controller. In our proposed method, we consider various aspects of PIC and PLC based power factor controller. The main core of this research work is to design a PIC based power factor controller. The proposed system has the ability to control the power factor of both linear and nonlinear load system.

\subsection{Power Factor Correction using PLC}

Seimens Controller PLC (S7-300) as shown in Figure 3 drives the PFC. System consists of following modules,

1) Power supply

2) $\mathrm{CPU}$

3) Digital inputs

4) Analog to digital convertor

The digital input module is a $12 \mathrm{VDC}, 13-30 \mathrm{~V}$ for ' 1 ' logic and -3 to $5 \mathrm{~V}$ for ' 0 ' logic with 6 input points. The module is used to read three different pulses from the signal conditioning circuit, which represents the phase shift between the voltage and current in each phase (i.e., $\left.\varphi_{a}, \varphi_{b}, \varphi_{c}\right)$. Another port of the digital input module is used to read the output of the zero crossing detectors (ZCD) circuit. Such information is used to switch the compensation capacitors $\mathrm{ON}$ or OFF at the zero crossing time to minimize the transient effect.

The digital output module is a 6 points, 0.5 Amps output current, 12 VDC rated load voltage. It is used to switch the capacitors that compensates for the power factor based on the decision made by the control strategy (written as software in the PLC). The decision is developed according to the measurement of the phase shift, which is introduced via the input module. The first one of the two transducers is an ac-to-dc transducer (Vac-to-Vdc) for the phase voltage while the second is a frequency to voltage transducer (F-to-Vdc) for the stator frequency. These two signals are used to maintain constant ratio of voltage to-frequency (Voltage/f) during the power factor correction process. The voltage transducer accepts an input analog signal in the range of $01-50 \mathrm{~V} \mathrm{AC}$ and gives an output of 1-5V DC (direct current) though $5 \mathrm{~K} \Omega$. On the other hand, the frequency transducer accepts input single in the range of $55-65 \mathrm{~Hz}$ with a voltage of $120 \mathrm{~V} \mathrm{AC} 10 \%$ and gives $0-5 \mathrm{~V}$ DC through a load of $5 \mathrm{~K} \Omega$.

\subsection{PLC Comparison with Control Systems}

PLCs are well adapted to a range of automation tasks. These are typically industrial processes in manufacturing where the cost of developing and maintaining the automation system is high relative to the total cost of the automation, and where changes to the system would be expected during its operational life. PLCs contain input and output devices compatible with industrial pilot devices and controls; little electrical design is required, and the design problem centers on expressing the desired sequence of operations. PLC applications are typically highly customized systems so the cost of a packaged PLC is low compared to the cost of a specific custom-built controller design [1]

On the other hand, in the case of mass-produced goods, customized control systems are economic due to the lower cost of the components, which can be optimally chosen instead of a "generic" solution, and where the non-recurring engineering charges are spread over thousands or millions of units $[13,16]$. For high volume or very simple fixed automation tasks, different techniques are used [8, 11]. For example, an electromechanical cam timer costing only a few dollars in production quantities would control a consumer dishwasher.

A microcontroller-based design would be appropriate where hundreds or thousands of units are produced and so the development cost (design of power supplies, input/output hardware and necessary testing and certification) can be spread over many sales, and the end-user would not need to alter the control. Automotive applications are an example; millions of units are built each year, and very few end-users alter the programming of these controllers. However, some specialty vehicles such as transit busses economically use PLCs instead of custom-designed controls; because of low volumes the development cost is uneconomic. Very complex process control, such as used in the chemical industry, may require algorithms and performance beyond the capability of even high-performance PLCs.

\subsection{Demerits of CPFC and its Solution}

Capacitor selection is a big problem in capacitive power factor control [7, 8]. Static Power factor correction must neutralize no more than $80 \%$ of the magnetizing current of the motor. If the correction is too high, there is a high probability of over correction, which can result in equipment failure with severe damage to the motor and capacitors. Unfortunately, the magnetizing current of induction motors varies considerably between different motor designs. The magnetizing current is almost always higher than $20 \%$ of the rated full load current of the motor, but can be as high as $60 \%$ of the rated current of the motor. Most power factor correction is too light due to the selection based on tables, which have been published by a number of sources [4]. These tables assume the lowest magnetizing current and quote capacitors for this current. In practice, this can mean that the correction is often less than half the value that it should be and the consumer is unnecessarily penalized.

Power factor correction must be correctly selected based on the actual motor being corrected. The electrical calculations software provides two methods of calculating the correct value of KVAR correction to apply to a motor. The first method requires the magnetizing current of the motor. For the availability of magnetizing current figure, this is the preferred method. Where the magnetizing current is not available, the second method is employed and is based on the half load power factor and efficiency of that motor. Supply harmonics on the supply cause a higher current to flow in the capacitors $[3,15]$. This is because the impedance of the capacitors gets down as the frequency increases. This 
increase in current flow through the capacitor results in additional heating of the capacitor and reduces its life. The harmonics are caused but many nonlinear loads, the most common in the industrial market today, are the variable speed controllers and switch mode power supplies.

Harmonic voltages can be reduced by the use of a harmonic compensator, which is essentially a large inverter that cancels out the harmonics. This is an expensive option. Passive harmonic filters comprising resistors, inductors and capacitors can also be used to reduce harmonic voltages. This is also an expensive exercise. In order to reduce the damage caused to the capacitors by the harmonic currents, it is becoming common today to install detuning reactors in series with the power factor correction capacitors [7]. These reactors are designed to make the correction circuit inductive to the higher frequency harmonics. Typically, a reactor would be designed to create a resonant circuit with the capacitors above the third harmonic, but sometimes it is below. Adding the inductance in series with the capacitors reduces their effective capacitance at the supply frequency. Reducing the resonant or tuned frequency will reduce the effective capacitance further. The object is to make the circuit look as inductive as possible at the $5^{\text {th }}$ harmonic and higher, but as capacitive as possible at the fundamental frequency. Detuning reactors will also reduce the chance of the tuned circuit formed by the capacitors and the inductive supply being resonant on a supply harmonic frequency, thereby reducing damage due to supply resonances amplifying harmonic voltages caused by non linear loads.

Detuning reactors are connected in series with power factor correction capacitors to reduce harmonic currents and to ensure that the series resonant frequency does not occur at a harmonic of the supply frequency. The reactors are usually chosen and rated as either $5 \%$ or $7 \%$ reactors. This means that at the line frequency, the capacitive reactance is reduced by $5 \%$ or $7 \%$. Using detuning reactors results a lower KVAR, so the capacitance needs to be increased for the same level of correction. When detuning reactors are used in installations with high harmonic voltages, there can be a high resultant voltage across the capacitors. This necessitates the use of capacitors that are designed to operate at a highly sustained voltage. Capacitors designed for use at line voltage only, should not be used with detuning reactors. Check the suitability of the capacitors for use with line reactors before installation.

The detuning reactors can dissipate a lot of heat. The enclosure must be well ventilated, typically forced air-cooled. The detuning reactor must be specified to match the KVAR of the capacitance selected. The reactor would typically be rated as $12.5 \mathrm{KVAR} 5 \%$ meaning that it is a $5 \%$ reactor to connect to a $12.5 \mathrm{KVAR}$ capacitor. Reactive power in a DC circuit or in an AC circuit whose impedance is a pure resistance, the voltage and current are in phase, and the following equation holds;

$$
P=E_{r m s} I_{r m s}
$$

where $\mathrm{P}$ is the power is watts, $E_{r m s}$ is the root mean square (rms) voltage and $I_{r m s}$ is the rms current in amperes but in an AC circuit whose impedance consists of reactance as well as resistance, the voltage and current are not in phase. This complicates the determination of power. In the absence of reactance, the product $E_{r m s} I_{r m s}$ represents the true power but when there is reactance in an AC circuit, the product $E_{r m s} I_{r m s}$ is greater than the true power. The excess is called reactive power, and represents energy alternately stored and released by inductors and/or capacitors. The vector sum of the true and reactive power is known as apparent power.

\section{Proposed Design Implementation}

In the working of this system it is mainly divided in to three sections that are power factor detection section, PLC section for the switching of the capacitor bank and capacitor bank section for the improvement of the power factor.

\subsection{Zero Crossing Detector}

ZCD circuits consist of two independent, high gains, internally frequency-compensated, which were designed specifically to operate from a single power supply over a wide range of voltages [1]. The circuit schematic diagram is shown in Fig 2. The low power supply drain is independent of the magnitude of the power supply voltage. Application areas include transducer amplifiers, DC gain blocks and all the conventional op-amp circuits which now can be more easily implemented in single power supply systems. For example, these circuits can be directly supplied with the standard $+5 \mathrm{~V}$, which are used in logic. Such systems easily provide the required interface with electronics without requiring any additional power supply. In the linear mode the input common-mode voltage range includes ground and the output voltage can also swing to ground, even though operated from only a single power supply voltage. 

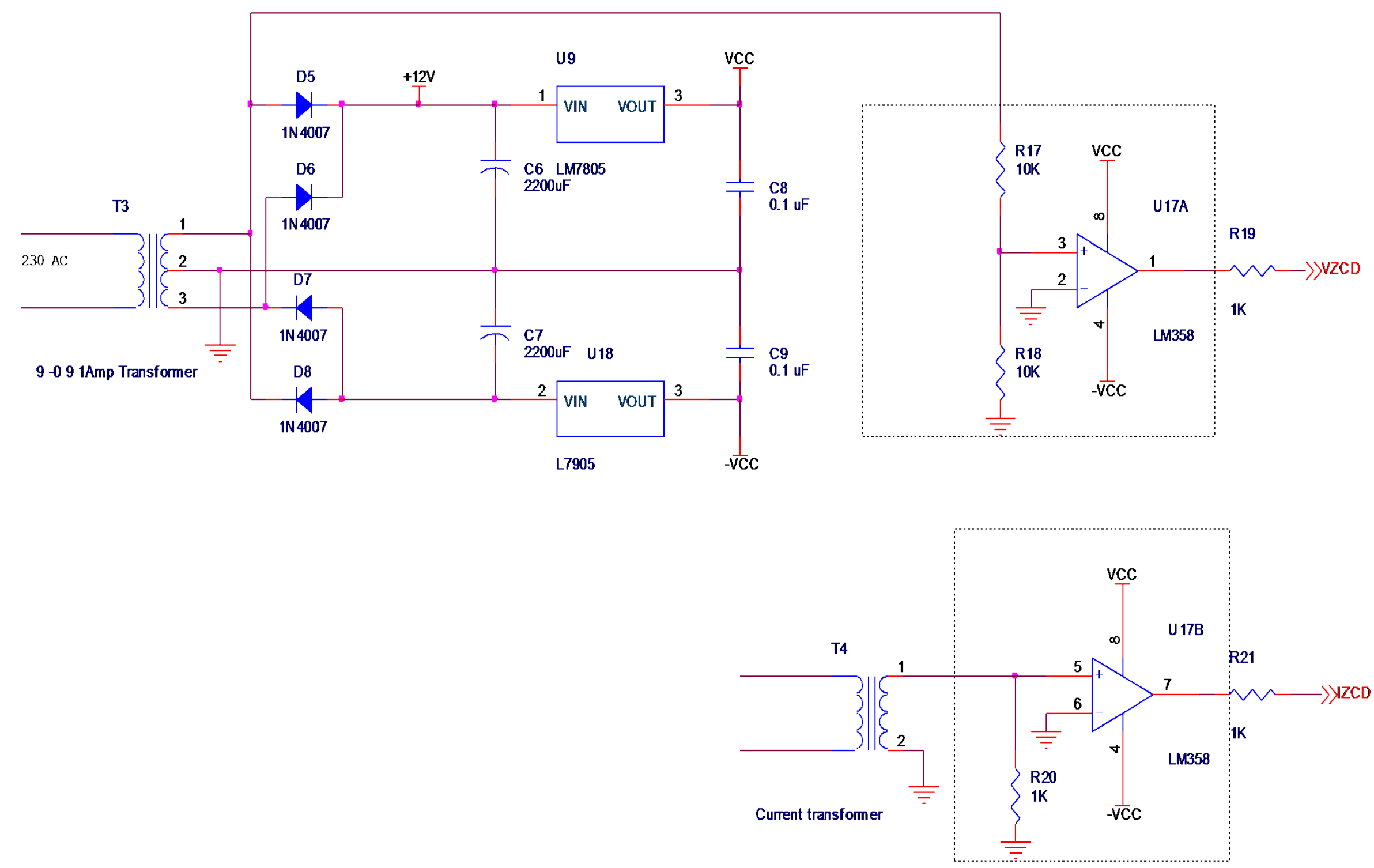

Figure 2. Schematic of ZCD 


\subsection{Power Factor Detection Unit}

In this section the major components are: current transformer, potential transformer, PIC microcontroller and LCD display. The potential transformer connected parallel to the supply and the current transformer connected series to the supply line. Both components step down the supply voltage and current to desirable values, which are given as input to the ZCD. ZCD convert both sine waves into the corresponding square waves. If the phase difference were 90-degree power factor would be zero. For 90 degree the time delay is 5 milliseconds.

From that we generated a count value above that value the power factor is a leading one in between that by using the count value the angle and power factor is calculated. The programming of PIC is done on the micro controller. The count value, angle and power factor are shown on the LCD display. For the PIC timer 1 is used for this purpose because it's the only 16-bit timer. The PIC works in the capture mode in order to find out the phase difference by continuously capturing the square waveforms from the ZCD. We get two outputs from the PIC and give the outputs to the PLC unit whether the power factor is leading or lagging. LCD is interfaced with the PIC microcontroller.

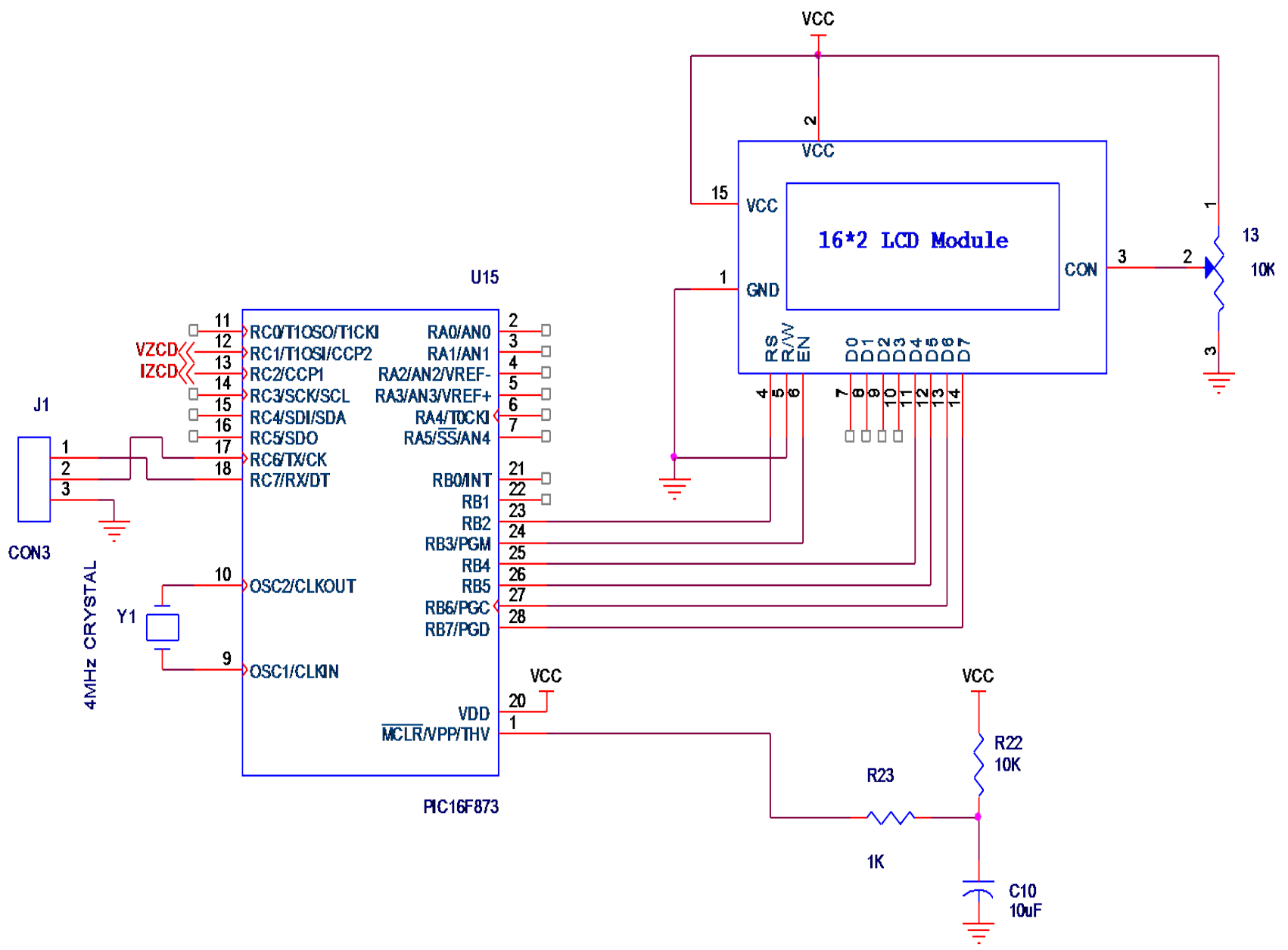

Figure 3. PIC and LCD interface circuit.

\subsection{Capacitor Bank Unit}

The capacitor banks are switched with the help of the relays. In the capacitor bank section capacitors with different values are present. But in our project we are using two capacitor units for the power factor improvement. According to the output of PLC the capacitors get switched ON. First if the power factor is lagging the $\mathbf{C} 1$ is switched ON then check for the power factor value and then switched ON C2 and then both capacitors 1 and 2 respectively. If any leading power factor came both the capacitor 1 and 2 switched OFF and repeat from the initial state. 


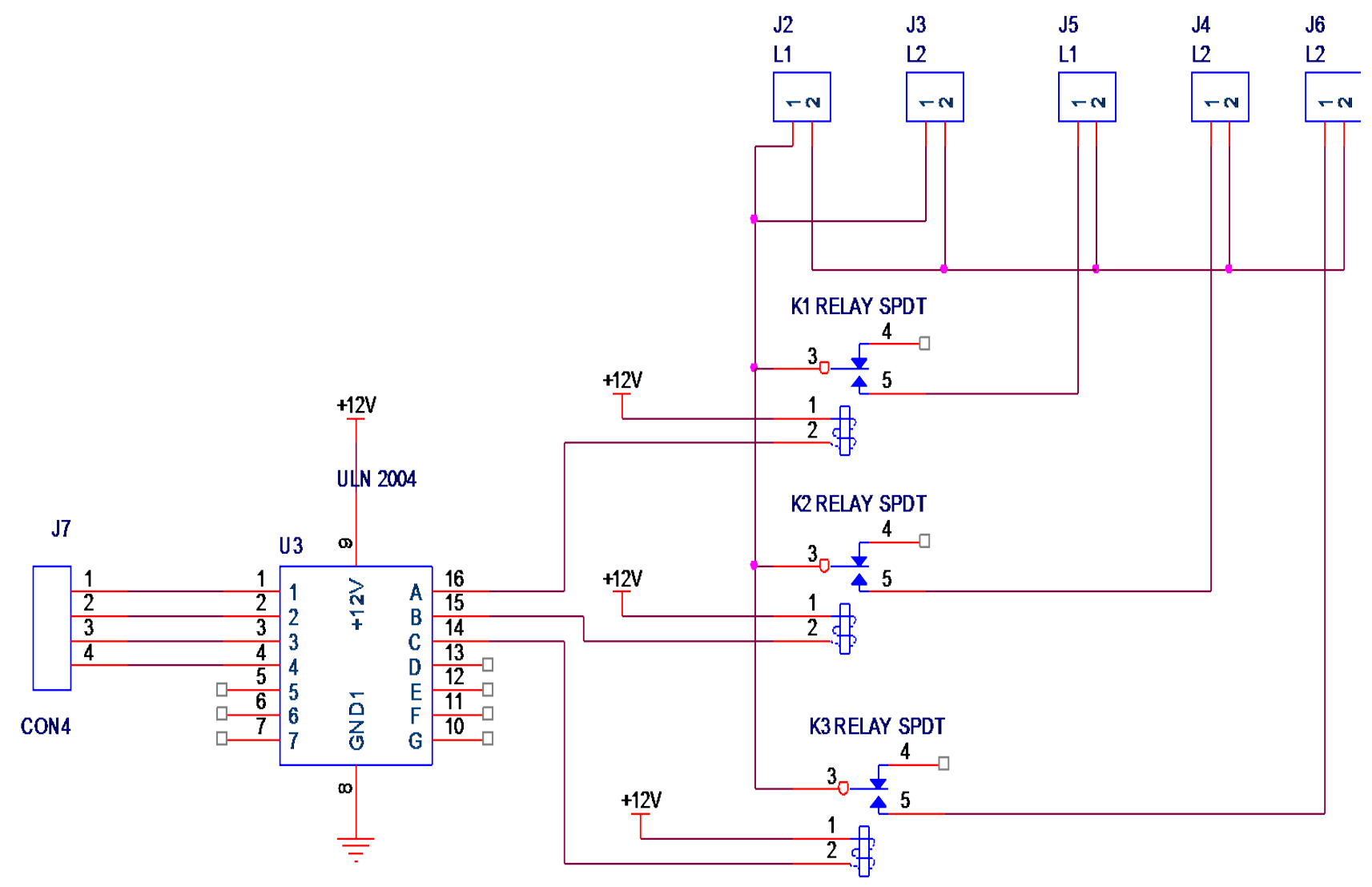

Figure 4. Capacitor Bank Unit

\subsection{Motor Control}

The speed of the motor is controlled through VSD circuit. We use MOC 3020, which acts as a dimmer for controlling the speed of the motor. The speed of the motor is controlled through two push buttons. First button increases the speed while the other one decreases it. The hardware of the project is given below,
1) Integrated circuit (IC) LM-735
2) MOC 3020
3) PIC18F452 controller
4) Light dependent resistor (LDR)
5) Light emitting diode (LED)
6) Resistors and capacitors.

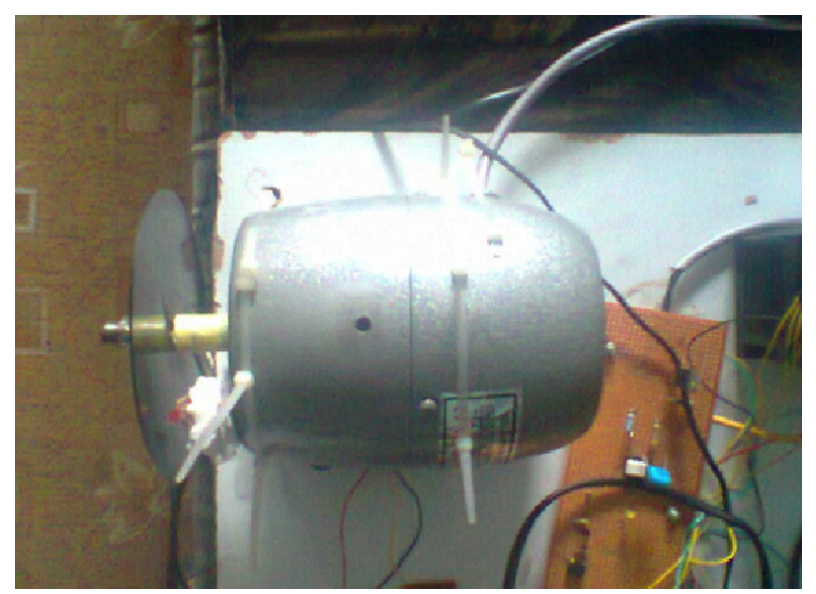

Figure 5. Single phase Induction Motor.

\section{Practical Implementation and Testing}

\subsection{Practical Implementation}

The practically implemented and complete system hardware is shown in Fig. 5, 6, 7, 8 and 9.

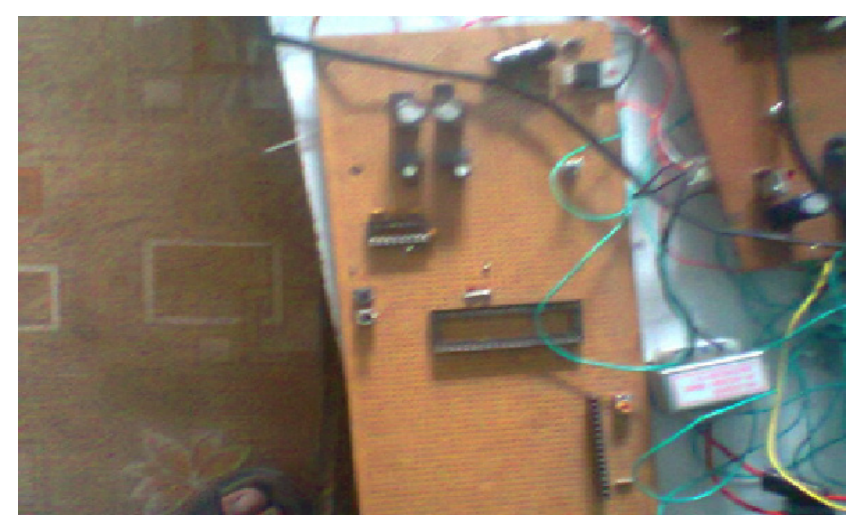

Figure 6. Motor controlling Unit. 


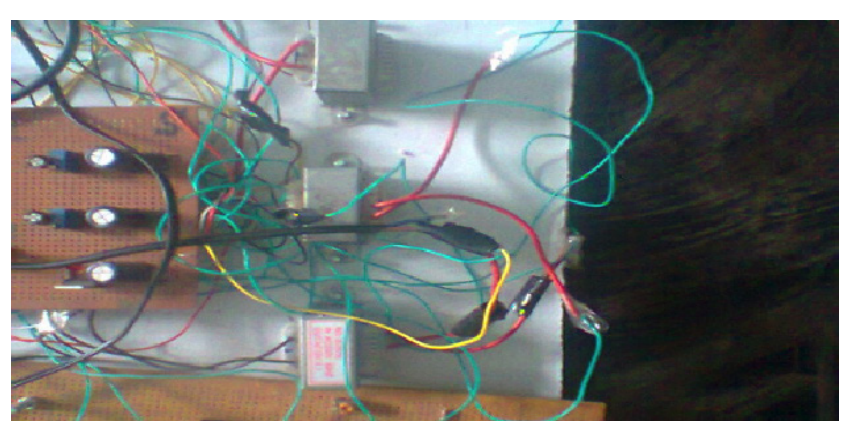

Figure 7. Power supply with $\mathrm{CT}$ and PT.

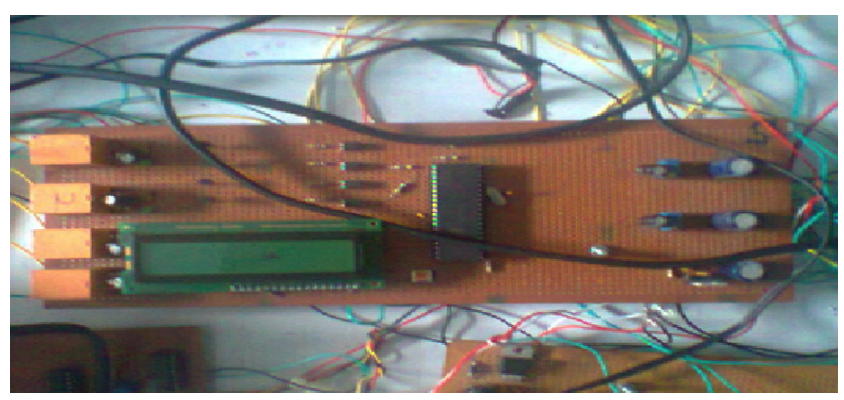

Figure 8. Relay section.

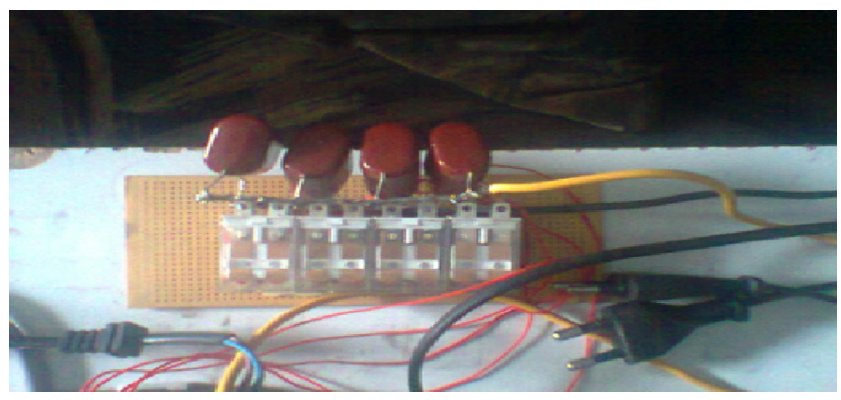

Figure 9. Capacitor bank unit.

\subsection{Testing}

When we started our project the first problem that we faced is that, PLC is a digital device and our input to PLC was analogue. So first we decided to design three analogues to digital converter (ADC) modules but this was not a feasible solution. From the literature [1], the use of microcontroller was recommended to control the motor instead of using ADC modules. We choose PIC18546 that has built-in ADC converter. In the beginning, our idea was to control the motor and using the PLC controlled the power factor of motor. By doing this we eliminated the problem of analogue to digital conversion.

When first we used the microcontroller and did it's soldering on a circuit board and run it for operation it burnt out. The issue was analyzed and we did complete measurements of current and potential transformers. It was found that transformers were over rated for the operation of microcontroller. Then we properly designed the transformers and our microcontroller began working properly. Then we control the motor in a proper way.

The next task was to relate the power factor with the speed of the motor. At the start, when we connected the capacitor bank in parallel with the motor. The value of the power factor drops, which closes the relays and capacitors, provided the apparent power. The speed of motor increases due to the above mentioned abnormalities. We trouble shoot the issue by disconnecting the capacitors from motor and installed them at the distribution end of our main power supply. By the end of the day, we successfully improve the power factor of the motor.

\section{Conclusion and Future Recommendations}

We propose a flexible technique for power factor improvement. We see that the propose method well adapted by providing best power factor regarding the load. The proposed work can be extended to higher level by introducing the multiple and large motors. The PLC in our project belongs to FATEK PLC. We hardwired the circuit of the PLC and made an educational purpose PLC with low cost. Higher-level technical institutes and industries can purchase our low cost project for the practical purpose. Our proposed system can improve the lagging power factor up to 0.90 and the system remains safe from the different disadvantages of lagging power factors. By using the proposed system the power factor control becomes very fast and accurate than other methods. The electric charges can also be reduced with our system.

\section{Acknowledgements}

The authors would like to thank Prof. Noor M. Sheikh from Al-Khawarzami Research center, University of Engineering and Technology Lahore, Pakistan for the thorough discussion and providing useful suggestions during the course of investigation. Without great financial support of Innovative Private Limited, Lahore Pakistan, it would not be possible to carry out the simulations. The whole project during the program would have been nothing without the enthusiasm and imagination of our faculty members.

\section{REFERENCES}

[1] E. Thornton and J. Armintor, "The fundamentals of ac electric induction motor design and application," in Proc of the Twentieth International Pump Users Symposium, pp. 95 106, 2003.

[2] J. Ware, "Power Factor Correction," IEE Wiring Matters, Spring 2006: 1-3.

[3] B. S. Mandal, R., A. Kar, and S. P. Chowdhury, "A microcomputer-based power factor controller," IEEE Transaction on Industrial Electronics, vol. 41, no. 3, pp. 361-371, 1994. 
[4] Daut, I.; Irwan, Y.; Irwanto, M.; Safwati, I.; Gomesh, N.; Ezzani, M.; Risnidar, C.B., "Improvement of induction machine performance using power factor correction," 2011 International Conference on Electrical, Control and Computer Engineering (INECCE), pp: 472 - 476, 21-22 June 2011.

[5] N. Catalogue, Power Factor Correction. Electrical Engineering Products Pvt. LTD.: Industrial Switchgear and Automation Specialist (NHP), 2007.

[6] L. C. M. Y. Xiao, J. and J. Xie, "Lighting control and its power management in railway passenger station," in Asia Pacific power and energy engineering conference, 2009 (APPEEC'09), pp. 1 - 4, 2009.

[7] V.K. Mehta, "Power Factor Improvement"” National Certificates in Technology, 2005.

[8] N.A. Ahmed and E.H. El-Zohri, "Power factor improvement of single phase ac voltage controller employing extinction angle control technique," IEEE $46^{\text {th }}$ Symposium on Circuits and Systems, vol.3, pp: 1075-1080, 27-30 Dec. 2003.

[9] G. Venkataratnam, K. Ramakrishnaprasad and N. Yadaiah, "P.F. Correction of 3-Ø induction motor drive through PWM current controlled technique," Internation Journal of Computer and Electrical Engineering, vol. 3, no. 2, April 2012: 236-241.

[10] Q. Li and J.J. Cathey, "Input power factor correction, variable speed, single phase induction motor suitable for retrofit applications," Internation Journal of Electronics, vol. 93, no. 3, March 2006: 149-165.
[11] O. Semiconductor, Power Factor Correction (PFC) Handbook, 4th ed. Electrical Engineering Products Pvt. LTD.: Enabling Energy Efficient Solutions, 2011.

[12] Dimitrov L. Rossetto, G. Spiazzi, and T. P., "Control Techniques for Power Factor Correction Converters," IEEE Transaction on Industrial Electronics, vol. 41, no. 3, pp. 1-9, Aug. 1994.

[13] R. Redl, "Power Factor Correction in Single-Phase Switching-mode Power Supplies - An Overview," International Journal of Electronics on switching mode power supplies, vol. 4, no. 2, pp. 1-10, May 1992.

[14] Heger, C.A. and Sen, P. K. and Morroni, A. "Power factor correction \#x2014; A fresh look into today's electrical systems," 2012 IEEE-IAS/PCA 53rd Cement Industry Technical Conference, pp: 1-13, 2012.

[15] Kanaan, H.Y. and Somers, C. and Al-Haddad, K., "Power Factor Correction with a Modified Sheppard-Taylor Topology Operating in Discontinuous Capacitor Voltage Mode and Low Output Voltage," IEEE Journal of Emerging and Selected Topics in Power Electronics, 2014, PP(99): 1-1.

[16] Ramezan Ghanbari, A. and Adib, E. and Farzanehfard, H., "Single-stage single-switch power factor correction converter based on discontinuous capacitor voltage mode buck and flyback converters," IET Power Electronics, 2013, 6(1): $146-152$. 